\title{
Stochastic Biasing of Galaxies and Dark Halos in Cosmological Hydrodynamic Simulations
}

\author{
Kohji Yoshikawa \\ Department of Astronomy, Kyoto University
}

\begin{abstract}
We perform an extensive analysis of nonlinear and stochastic biasing of galaxies and dark halos in spatially flat low-density CDM universe using cosmological hydrodynamic simulations. We identify galaxies by linking cold and dense gas particles which satisfy the Jeans criterion. We compare their biasing properties with the predictions of an analytic halo biasing model. Dark halos in our simulations exhibit reasonable agreement with the predictions only on scales larger than $10 \mathrm{Mpc} / \mathrm{h}$, and on smaller scales the volume exclusion effect of halos due to their finite size becomes substantial. Interestingly the biasing properties of galaxies are better described by extrapolating the halo biasing model predictions.
\end{abstract}

\section{Introduction}

One of the most important goals of ongoing galaxy redshift surveys like Sloan Digital Sky Survey (SDSS), is to reveal the large scale structure of the universe from the distribution of visible galaxies. Since the theories for dark matter clustering are well established (Peacock \& Dodds 1996) and confirmed using numerical simulations (Suto 1993; Navarro et al. 1997), we are ready to probe the cosmological model and the properties of primordial density fluctuation once we have the clustering properties of dark matter. However, it is widely believed that the distribution of galaxies are somewhat biased relative to the underlying dark matter density field, since the formation of galaxies is subject to many complicated physical processes other than gravitational dynamics. The observational results such as morphology dependent clustering of galaxies (Dressler et al. 1980;Loveday et al. 1995) and the clustering of Lyman-break galaxies (Steidel et al. 1998) also indicate the galaxy biasing is sensitive to many physical processes and thus stochastic. Therefore, the relation between galaxy and dark matter clustering is far from simple and the primary difficulty in properly interpreting the observational data of upcoming redshift surveys.

In this work, we use the cosmological hydrodynamic simulations (Yoshikawa et al. 2000) of galaxy formation in cold dark matter (CDM) universe and investigate the biasing properties of galaxies dark matter cores (DM cores) and dark halos. Particularly, we focus on the comparison of their biasing with the theoretical prediction of halo biasing by Taruya \& Suto (2000). 


\section{Numerical Simulations}

Our simulation code is a hybrid of Particle-Particle-Particle-Mesh $\left(\mathrm{P}^{3} \mathrm{M}\right)$ Poisson solver and smoothed particle hydrodynamics (SPH) algorithm. The simulation presented in this paper adopts $N_{\mathrm{DM}}=128^{3}$ dark matter particles and the same number of gas particles. We consider the spatially-flat low-density CDM (LCDM) universe with $\Omega_{0}=0.3, \lambda_{0}=0.7, \sigma_{8}=1.0$ and $h=0.7$, where $\Omega_{0}$ is the mean mass density parameter, $\lambda_{0}$ the dimensionless cosmological constant, $\sigma_{8}$ the rms density fluctuation on a scale of $8 h^{-1} \mathrm{Mpc}$, and $h$ the Hubble constant in the units of $100[\mathrm{~km} / \mathrm{s} / \mathrm{Mpc}]$. We assume the mean baryon density parameter to be $\Omega_{\mathrm{b}}=0.015 h^{-2}$. The simulation is carried out with a periodic cube of $\left(75 h^{-1} \mathrm{Mpc}\right)^{3}$, with the gas and dark matter mass per particles being $2.4 \times 10^{9} M_{\odot}$ and $2.2 \times 10^{10} M_{\odot}$, respectively.

\section{Biasing properties of galaxies, dark matter cores and dark halos}

Galaxies in our simulation are identified as clumps of cold and dense gas particles which satisfy the Jeans criterion $h_{\mathrm{SPH}}>c_{\mathrm{s}} / \sqrt{\pi G \rho_{\text {gas }}}$ and $\rho_{\text {gas }}>10^{2} \bar{\rho}_{\mathrm{b}}(z)$, where $h_{\mathrm{SPH}}$ is the smoothing length of gas particles, $G$ the gravitational constant, $\rho_{\text {gas }}$ the local gas density, and $\bar{\rho}_{\mathrm{b}}(z)$ the mean baryon density at redshift $z$. We extract the clumps using friend-of-friend (FOF) method with linking length $b=0.0164(1+z)$ times mean gas particle separation. Dark halos are also identified using FOF algorithm with linking length $b=0.164$ times mean dark matter particle separation. From the distributions of dark halos, and galaxies, we compute the number density fields of each objects $\delta_{i}\left(\mathbf{x}, R_{s}\right)$ where the subscript "g" and " $h$ " represents galaxies, dark halos, respectively, smoothed over the tophat window radius $R_{s}$. We find that the simulated dark halos exhibit reasonable agreement with the predictions for small $\delta_{\mathrm{m}}$. On the other hand, they tend to be underpopulated for large $\delta_{\mathrm{m}}$ or anti-biased. This is mainly because of the volume exclusion effect of halos due to their finite size. Since galaxies according to our definition have smaller extent than dark halos, they do not suffer from the volume exclusion effect so seriously. In fact, the simulated biasing of galaxies shows much better agreement with the theoretical predictions despite the fact that the models are formally valid only for dark halos defined according to the Press-Schechter manner.

\section{References}

Peacock, J.A. \& Dodds, S.J. 1996, MNRAS, 280, L19

Suto, Y. 1993, Prog.Theor.Phys. 90, 117

Navarro, J., Frenk, C. \& White, S.D.M. 1997, ApJ, 490, 493

Dressler, A. 1980, ApJ, 236, 351

Loveday, J., Maddox, S.J., Efstathiou, G. \& Peterson, B. A. 1995, ApJ, 442, 457

Steidel, C.C., Adelberger, K.L., Dickinson, M., Giavalisco, M., Pettini, M. \& Kellogg, M. 1998, ApJ, 492, 428

Yoshikawa, K., Jing, Y.P. \& Suto, Y. 2000, ApJ, 535, 593

Taruya, A. \& Suto, Y. 2000, ApJ, 542, 559 (TS) 